\title{
A CONVERGENCE THEOREM FOR SINGULAR INTEGRAL EQUATIONS
}

\author{
DAVID ELLIOTT
}

(Received 4 June 1980)

(Revised 2 December 1980)

\begin{abstract}
The principal result of this paper states sufficient conditions for the convergence of the solutions of certain linear algebraic equations to the solution of a (linear) singular integral equation with Cauchy kernel. The motivation for this study has been the need to provide a convergence theory for a collocation method applied to the singular integral equation taken over the arc $(-1,1)$. However, much of the analysis will be applicable both to other approximation methods and to singular integral equations taken over other arcs or contours. An estimate for the rate of convergence is also given.
\end{abstract}

\section{Introduction}

We shall take as the singular integral equation of the title the equation defined on $-1<t<1$ by

$$
a(t) \phi(t)+\frac{b(t)}{\pi} f_{-1}^{1} \frac{\phi(\tau) d \tau}{\tau-t}+\int_{-1}^{1} k(t, \tau) \phi(\tau) d \tau=y(t)
$$

The real functions $a, b, k$ and $y$ are given and we require the unknown function $\phi$. Following Muskhelishvili [7] we shall assume that $a, b$ and $y$ are Hölder continuous on $[-1,1] ; k$ is assumed to be Hölder continuous on $[-1,1] \times$ $[-1,1]$. The Cauchy principal value integral $f_{-1}^{1}(\phi(\tau) /(\tau-t)) d \tau$ is defined by

$$
\lim _{e \rightarrow 0+}\left(\int_{-1}^{t-e}+\int_{t+e}^{1}\right)(\phi(\tau) /(\tau-t)) d \tau
$$

OCopyright Australian Mathematical Society 1981 
and we look for solutions $\phi$ of (1.1) in the class of functions that Muskhelishvili denotes by $H^{*}[-1,1]$. That is, a function $g \in H^{*}[-1,1]$ if $g$ is Hölder continuous on every closed interval contained in $(-1,1)$ and is integrable at each end point. Following Dow and Elliott [1] we choose as a new dependent variable in place of $\phi$ the function $x$ defined by

$$
x=r \phi / Z \text { or } \phi=Z x / r \text {, }
$$

where $r=\left(a^{2}+b^{2}\right)^{1 / 2}$, it being assumed that $r(t)>0$ for $t \subset\{-1,1\}$ so thât (1.1) is of normal type. The function $Z$ of (1.2) is the so called fundamental function (see [7] and [1, defn. 2.6]) and it can be shown that $Z \in H^{*}[-1,1]$. It then follows that $x \in H[-1,1]$, the space of Hölder continuous functions on $[-1,1]$. In terms of $x$ we rewrite $(1.1)$ as $A x+K x=y$ where

$$
\begin{aligned}
& A x(t)=\frac{a(t) Z(t)}{r(t)} x(t)+\frac{b(t)}{\pi} f_{-1}^{1} \frac{Z(\tau) x(\tau) d \tau}{r(\tau)(\tau-t)}, \\
& K x(t)=\int_{-1}^{1}(Z(\tau) k(t, \tau) x(\tau) / r(\tau)) d \tau .
\end{aligned}
$$

It might be noted at this point that although we have chosen to consider the singular integral equation taken over the arc $(-1,1)$, most of the analysis which follows will be appropriate if (1.1) is taken over a closed contour $\mathcal{C}$, say. Equally well we could take $C$ to be a union of arcs and/or closed contours, but we shall not pursue this generalization any further in this paper. When $K \equiv 0$, the equation $A x=y$ will be referred to as the dominant equation; otherwise the equation $(A+K) x=y$ is known as the complete equation.

In order to find approximate solutions of (1.1) we must first discretize it in some way so that it is replaced by a sequence of linear algebraic equations each member, of which can be written as $\left(A_{n}+K_{n}\right) x_{n}=y_{m}$ where $A_{n}, K_{n}$ denote $m \times n$ matrices, $x_{n}$ is an $n \times 1$ column vector and $y_{m}$ is an $m \times 1$ column vector. The relationship between $m$ and $n$ will be made more precise later. The purpose of the convergence theory is to provide sufficient conditions on $A_{n}$ and $K_{n}$ so that the vectors $x_{n}$ converge, in some sense, to $x$ as $n \rightarrow \infty$. In Section 2 we shall state some selected results from the theory of singular integral equations which we shall require for our analysis. In Section 3 we first review briefly some results from the theory of linear algebraic equations and then introduce the so called restriction and prolongation operators which are needed to relate $x_{n}$ to $x$. The convergence of approximate solutions of the dominant equation is discussed in Section 4 and finally, in Section 5, we give a convergence theorem for the complete equation.

It should be pointed out that, in one sense, much of what follows can be said to be known. The operator $A$ is known as a Fredholm (or Noether) operator and a convergence theory which includes such operators has been given by Stummel 
[10]. (The author wishes to thank a referee for bringing this and other references to his attention.) Nevertheless it appears to be a useful exercise to exhibit a convergence theory which is directly related to the particular context in which it is required. There appears to be at present a growing interest in finding approximate solutions to equations such as (1.1) and one can attempt to apply Theorem 5.5 to any approximation scheme which may be proposed for solving (1.1). An example of an application of this theorem has already been given by the author [3].

\section{The theory of singular integral equations}

In order to keep this section within reasonable bounds, proofs will not be given but may be obtained from results in the standard texts (see [7], [4]). From a knowledge of $a$ and $b$ we first determine the index $\kappa$ of (1.1), (see [1]). The index $\kappa$ is an integer (positive, negative or zero) which determines the form of the solution of (1.1). Thus, when $\kappa>0$ we find that the null space $\operatorname{of} A, \operatorname{ker}(A)$, is of dimension $\kappa$ and is spanned by the functions $\left\{b, t b, t^{2} b, \ldots, t^{\kappa-1} b\right\}$. In order to see the significance of $\kappa<0$ it is convenient at this point to introduce the operator $A^{*}$ which is the adjoint of $A$ and is defined by

$$
A^{*} \psi(t)=\frac{a(t) Z(t)}{r(t)} \psi(t)-\frac{Z(t)}{r(t)} \cdot \frac{1}{\pi} f_{-1}^{1} \frac{b(\tau) \psi(\tau)}{\tau-t} d \tau,
$$

for $-1<t<1$. The operator $A^{*}$ is such that for any $\psi_{1}, \psi_{2} \in H[-1,1]$ we have

$$
\int_{-1}^{1} \psi_{1}(t) A \psi_{2}(t) d t=\int_{-1}^{1} \psi_{2}(t) A^{*} \psi_{1}(t) d t
$$

The null space of $A^{*}$ is such that $\operatorname{dim} \operatorname{ker}\left(A^{*}\right)=\max (-\kappa, 0)$ and when $\kappa<0$, $\operatorname{ker}\left(A^{*}\right)$ is spanned by the functions $\left\{1 / r Z, t / r Z, \ldots, t^{-\kappa-1} / r Z\right\}$. Since $\operatorname{dim} \operatorname{ker}(A)=\max (\kappa, 0)$ we see that $\kappa=\operatorname{dim} \operatorname{ker}(A)-\operatorname{dim} \operatorname{ker}\left(A^{*}\right)$.

To find the solution of (1.1) it is convenient at this point to introduce the operator $\hat{A}^{l}$ which is a sort of inverse of $A$. We define for $-1<t<1$

$$
\hat{A}^{\prime} y(t)=\frac{a(t) y(t)}{r(t) Z(t)}-\frac{b(t)}{\pi} f_{-1}^{1} \frac{y(\tau) d \tau}{r(\tau) Z(\tau)(\tau-t)} .
$$

The explicit relationship between $A$ and $\hat{A}^{\prime}$ is given as follows: When $\kappa \geqslant 0$ we have

$$
\left.\begin{array}{ll}
A \hat{A}^{\prime} y=y, & \text { for all } y \in H[-1,1], \\
\hat{A}^{\prime} A x=x+x^{(0)}, & \text { for all } x \in H[-1,1],
\end{array}\right\}
$$


where $x^{(0)}$ is an element of $\operatorname{ker}(A)$ depending on $x$. When $\kappa<0$ we have

$$
\left.\begin{array}{ll}
\hat{A}^{I} A x=x, & \text { for all } x \in H[-1,1], \\
A \hat{A}^{I} y=y+y^{(0)}, & \text { for all } y \in H[-1,1],
\end{array}\right\}
$$

where $y^{(0)}$ is now some element of $\operatorname{ker}\left(\hat{A}^{\prime}\right)$. Thus we see that $\hat{A}^{\prime}$ is a right-inverse of $A$ when $\kappa \geqslant 0$ and a left-inverse when $\kappa<0$; only when $\kappa=0$ is $\hat{A}^{I}$ the inverse of $A$. For any vaiue of $\kappa$ the solution of the dominant equation $A x=y$ is given by

$$
\left.\begin{array}{ll}
x=\hat{A}^{\prime} y+b P_{\kappa-1}, & \text { provided that } \\
\int_{-1}^{1} \frac{\tau^{k-1} y(\tau)}{r(\tau) Z(\tau)} d \tau=0, & k=1(1)(-\kappa) .
\end{array}\right\}
$$

Here $P_{\kappa-1}$ denotes an arbitrary polynomial of degree $\leqslant \kappa-1$ and is taken to be identically zero when $\kappa \leqslant 0$. The second of equations (2.5) are the so-called consistency conditions which can also be expressed as requiring that $y$ is orthogonal to $\operatorname{ker}\left(A^{*}\right)$. When $\kappa \geqslant 0$, we can solve $A x=y$ for all $y \in H[-1,1]$ and we only have a restriction on $y$ when $\kappa<0$.

Let us consider now the complete equation. If we rewrite this as $A x=y-$ $K x$, consider the right hand side as known and apply the results of the preceding paragraphs we find

$$
\left.\begin{array}{ll}
x=\hat{A}^{l}(y-K x)+b P_{\kappa-1}, & \text { provided that } \\
\int_{-1}^{1} \frac{\tau^{k-1}(y(\tau)-K x(\tau))}{r(\tau) Z(\tau)} d \tau=0, & k=1(1)(-\kappa) .
\end{array}\right\}
$$

The first of (2.6) can be rewritten as

$$
x+\hat{A}^{l} K x=A^{l} y+b P_{\kappa-1}
$$

which turns out to be a Fredholm integral equation with a "weakly singular" kernel. The reduction of (1.1) to (2.7) is known as the process of regularization and we shall assume throughout that for a given element $g$, the Fredholm equation $x+\hat{A}^{I} K x=g$ possesses a unique solution; in other words that $\left(I+\hat{A}^{\prime} K\right)^{I}$ exists.

At this point it is convenient to look upon the equation $(A+K) x=y$ in an abstract setting. We shall consider $A$ and $K$ as linear operators mapping a Banach space $X$ into a Banach space $Y$. The domain of $A, \operatorname{dom}(A)$, will be assumed to be dense in $X$. If we write $X=\operatorname{ker}(A) \oplus X^{(1)}$ then $\operatorname{dom}(A)=\operatorname{ker}(A)$ $\oplus\left\{X^{(1)} \cap \operatorname{dom}(A)\right\}$ so that $A$ operating on $X^{(1)} \cap \operatorname{dom}(A)$ is one-to-one and onto $\operatorname{ran}(A)$. Consequently $\hat{A}^{\prime}$ is the inverse operator defined on $\operatorname{ran}(A)$ into $X^{(1)} \cap \operatorname{dom}(A)$. In order that the dominant equation $A x=y$ should possess a 
solution we require that $\operatorname{ran}(A)$ be closed in $Y$, but having chosen $X, Y$ (see below) this is merely a statement of the consistency conditions (see (2.5)).

There appear to have been two common choices of the spaces $X$ and $Y$ in the literature to date. One is to choose $X=C[-1,1]$, the space of all continuous functions on $[-1,1]$ equipped with the uniform norm $\|\cdot\|_{\infty}$ defined by

$$
\|g\|_{\infty}=\max _{t \in[-1,1\}}|g(t)|, \quad \text { for any } g \in C[-1,1] \text {. }
$$

Since there are functions $g \in C[-1,1]$ for which $\mathrm{Ag}$ does not exist we choose $\operatorname{dom}(A)$ to be the space of all Hölder continuous functions defined on $[-1,1]$. This space, when equipped with the uniform norm, is not a Banach space but it is dense in $C[-1,1]$. If we choose $Y$ to be the space of all Hölder continuous functions on $[-1,1]$ we can make $Y$ a Banach space by giving it the so-called Hölder norm. The Hölder norm $\|\cdot\|_{H_{\mu}}$ where $0<\mu \leqslant 1$, is defined by

$$
\|g\|_{H_{\mu}}=\|g\|_{\infty}+\sup _{\substack{t_{1} \neq t_{2} \\ t_{1}, t_{2} \in[-1,1]}} \frac{\left|g\left(t_{1}\right)-g\left(t_{2}\right)\right|}{\left|t_{1}-t_{2}\right|^{\mu}} .
$$

A second choice for $X, Y$ is to choose $X$ to be the Banach space of square integrable functions on $(-1,1)$ with respect to the weight function $Z / r$ and to choose $Y$ to be the Banach space of square integrable functions with respect to the weight function $1 / \mathrm{Zr}$. These are the natural spaces to choose when using Galerkin type methods for the approximate solution of (1.1); see [6] and [2].

\section{Discrete equations; restriction and prolongation operators}

Given the equation $(A+K) x=y$, where $A$ is of index $\kappa$, we must discretize this in some way to give a system of linear algebraic equations which behaves at least qualitatively in the first instance like the original equation. To achieve this let us first define the index of any $m \times n$ matrix to be simply $n-m$. Throughout the remainder of this paper whenever the integers $m, n$ are used we shall have

$$
n-m=\kappa,
$$

where $\kappa$ is the index of (1.1). Next we observe that if any $m \times n$ matrix $A_{n}$ has $\operatorname{rank}=\min (m, n)$ then $\operatorname{dim} \operatorname{ker}\left(A_{n}\right)=\max (\kappa, 0)$ and $\operatorname{dim} \operatorname{ker}\left(A_{n}^{T}\right)$ $=\max (-\kappa, 0)$. Again, for such a matrix $A_{n}$ we have that when $\kappa>0$ it possesses an infinity of right inverses and when $\kappa \leqslant 0$ it possesses an infinity of left inverses. If we denote any such inverse by $\hat{A}_{m}^{I}$ then $A_{n}, \hat{A}_{m}^{I}$ are related by equations similar to (2.4). In particulart we might note that for all $x_{n} \in X_{n}$ and any value of $\kappa$ we have

$$
\hat{A}_{m}^{I} A_{n} x_{n}=x_{n}+x_{n}^{(0)}
$$


where $x_{n}^{(0)}$ is an element of $\operatorname{ker}\left(A_{n}\right)$ which depends upon $x_{n}$. Finally if $A_{n}$ again denotes an $m \times n$ matrix with rank $=\min (m, n)$ then the equation $A_{n} x_{n}=y_{m}$ possesses a solution only if $y_{m}$ is orthogonal to $\operatorname{ker}\left(A_{n}^{T}\right)$, see for example [8, Theorem 10.22]. With these results in mind we shall discretize (1.1) by choosing, for each $n$, a system of (real) linear algebraic equations of the form

$$
\left(A_{n}+K_{n}\right) x_{n}=y_{m} \text {, }
$$

where $A_{n}$ is an $m \times n$ matrix of rank $=\min (m, n), K_{n}$ is an $m \times n$ matrix and $x_{n}, y_{m}$ are $n \times 1$ and $m \times 1$ column vectors respectively. Arguing as in Section 2 we have that (3.3) is equivalent to

$$
\left(I_{n}+\hat{A}_{m}^{\prime} K_{n}\right) x_{n}=\hat{A}_{m}^{\prime} y_{m}+x_{n}^{(0)},
$$

where $x_{n}^{(0)} \in \operatorname{ker}\left(A_{n}\right)$ and provided that $y_{m}-K_{n} x_{n}$ is orthogonal to $\operatorname{ker}\left(A_{n}^{T}\right)$.

So much for the qualitative aspects of the discrete system. We must now choose $A_{n}, K_{n}$ so that in some sense they are good approximations to $A, K$ respectively. More fundamentally we must relate the spaces $X_{n}, Y_{m}$ to $X, Y$ respectively where $X_{n}$ denotes the space of all $n \times 1$ column vectors and $Y_{m}$ that of all $m \times 1$ column vectors. To do this we introduce the so-called restriction and prolongation operators. A restriction operator $r_{n}$ maps $X$ into $X_{n}$, a prolongation operator $p_{n}$ maps $X_{n}$ into $X$, subject to the following conditions:

$$
\left.\begin{array}{c}
\sup _{n}\left\|r_{n}\right\| \leqslant r<\infty, \quad \sup _{n}\left\|p_{n}\right\|<p<\infty ; \\
\lim _{n \rightarrow \infty}\left\|r_{n} x\right\|=\|x\|, \quad \text { for all } x \in X ; \\
r_{n} p_{n}=I_{n} ; \\
\lim _{n \rightarrow \infty}\left\|p_{n} r_{n} x-x\right\|=0, \text { for all } x \in X .
\end{array}\right\}
$$

Examples of such operators are to be found in [9]. Similarly for the spaces $Y_{m}$ and $Y$ we introduce a restriction operator $s_{m}$ which maps $Y$ into $Y_{m}$, and a prolongation operator $q_{m}$ which maps $Y_{m}$ into $Y$ such that:

$$
\left.\begin{array}{c}
\sup _{m}\left\|s_{m}\right\| \leqslant s<\infty, \quad \sup _{m}\left\|q_{m}\right\| \leqslant q<\infty ; \\
\lim _{m \rightarrow \infty}\left\|s_{m} y\right\|=\|y\|, \quad \text { for all } y \in Y ; \\
s_{m} q_{m}=I_{m} ; \\
\lim _{m \rightarrow \infty}\left\|q_{m} s_{m} y-y\right\|=0, \text { for all } y \in Y .
\end{array}\right\}
$$

We can now give a precise meaning to the statement that the sequence $\left\{x_{n}\right\}$, with $x_{n} \in X_{n}, n=1,2,3, \ldots$, converges to an element $x \in X$.

Definition 3.1 (i). A sequence $\left\{x_{n}\right\}$, with $x_{n} \in X_{n}$, converges discretely to $x \in X$ if $\lim _{n \rightarrow \infty}\left\|r_{n} x-x_{n}\right\|=0$. 
(ii) A sequence $\left\{x_{n}\right\}$, with $x_{n} \in X_{n}$, converges globally to $x \in X$ if $\lim _{n \rightarrow \infty}\left\|x-p_{n} x_{n}\right\|=0$.

From (3.5) it is straightforward to show that discrete convergence implies global convergence, and vice-versa, so that we can talk loosely of the convergence of $\left\{x_{n}\right\}$ to $x$.

As we shall see in Section 4, we also need a more restrictive definition of convergence than that given by Definition 3.1. First we introduce the idea of an $\alpha$-convergent sequence.

Definition 3.2 (i). A sequence $\left\{a_{n}\right\}$ is said to be $\alpha$-convergent to an element $a$ if, for all $n \geqslant n_{0}$, there exist positive constants $C$ and $\alpha_{1}$, independent of $n$, such that $\left\|a_{n}-a\right\| \leqslant C n^{-\alpha_{1}}$.

(ii) If a sequence $\left\{a_{n}\right\}$ is $\alpha$-convergent to the zero element it is said to be $\alpha$-null.

In relation to elements out of the spaces $X_{n}$ and $X$ we now have the following definition.

Definition 3.3 (i). A sequence $\left\{x_{n}\right\}, x_{n} \in X_{n}$, is said to converge $\alpha$-discretely to an element $x \in X$ if the sequence $\left\{r_{n} x-x_{n}\right\}$ is $\alpha$-null.

(ii) $A$ sequence $\left\{x_{n}\right\}, x_{n} \in X_{n}$, is said to converge $\alpha$-globally to an element $x \in X$ if the sequence $\left\{x-p_{n} x_{n}\right\}$ is $\alpha$-null.

It is trivial to show that $\alpha$-global convergence implies $\alpha$-discrete convergence; for the converse to be true we need to modify (3.5)(iv) but shall not pursue this further here.

We now introduce two basic ideas concerning sequences of linear operators which are fundamental to the convergence analysis.

Definition 3.4 (i). Suppose $B, B_{n}, n=1,2,3, \ldots$, are linear operators such that $B: X \rightarrow Y$ and $B_{n}: X_{n} \rightarrow Y_{m}$. The sequence of operators $\left\{B_{n}\right\}$ is said to be $\alpha$-discretely consistent with $B$ on $G \subseteq \operatorname{dom}(B)$ if, for all $x \in G$, the sequence $\left\{\delta_{n}^{B} x\right\}$ is $\alpha$-null where

$$
\delta_{n}^{B} x=s_{m} B x-B_{n} r_{n} x .
$$

(ii) Analogously if $C, C_{m}, m=1,2,3, \ldots$, are linear operators such that $C$ : $Y \rightarrow X, C_{m}: Y_{m} \rightarrow X_{n}$ then $\left\{C_{m}\right\}$ is $\alpha$-discretely consistent with $C$ on $H \subseteq$ $\operatorname{dom}(C)$ if, for every $y \in H$, the sequence $\left\{\delta_{n}^{C} y\right\}$ is $\alpha$-null where

$$
\delta_{n}^{C} y=r_{n} C y-C_{m} s_{m} y .
$$


In addition to operators being consistent we must also introduce the idea of stability. The definition to be given next is suggested by the detailed analysis given in [3] for the case of classical collocation.

Definition 3.5. The sequence of linear operators $\left\{A_{n}\right\}$ where $A_{n}: X_{n} \rightarrow Y_{m}$ is said to be $\alpha$-stable if, for each $n \geqslant n_{0}$, there exists a linear operator $\hat{A}_{m}^{I}: Y_{m} \rightarrow X_{n}$ such that $\left\|\hat{A}_{m}^{\prime}\right\| \leqslant C_{1}+C_{2} \log n$, whers $C_{1}, C_{2}$ are positive consiants independent of $n$.

With this definition we observe that if the sequence $\left\{\varepsilon_{m}\right\}, \varepsilon_{m} \in Y_{m}$, is $\alpha$-null then the sequence $\left\{\hat{A}_{m}^{l} \varepsilon_{m}\right\}$ is also $\alpha$-null.

In the next section we shall consider convergence of approximate solutions of the dominant equation; an analysis of the complete equation will be given in Section 5 .

\section{Convergence of approximate solutions of the dominant equation}

We can now give a convergence theorem for the dominant equation.

THeORem 4.1. Consider the equations $A x=y$, where $y$ is orthogonal to $\operatorname{ker}\left(A^{*}\right)$, and $A_{n} x_{n}=y_{m}$, where $y_{m}$ is orthogonal to $\operatorname{ker}\left(A_{n}^{T}\right)$. If

(i) $\left\{A_{n}\right\}$ is $\alpha$-discretely consistent with $A$ on $\operatorname{dom}(A)$,

(ii) $\left\{\boldsymbol{A}_{n}\right\}$ is $\alpha$-stable,

(iii) $\left\{y_{m}-s_{m} y\right\}$ is $\alpha$-null,

then to each solution $x$ of $A x=y$ there exists a solution $x_{n}$ of $A_{n} x_{n}=y_{m}$ such that $\left\{x_{n}\right\}$ converges $\alpha$-discretely to $x$.

Proof. From $A_{n} x_{n}=y_{m}$ we have $x_{n}=\hat{A}_{m}^{\prime} y_{m}+x_{n}^{(0)}$ where $x_{n}^{(0)}$ is any element of $\operatorname{ker}\left(A_{n}\right)$. On using (3.2) we have

$$
x_{n}-r_{n} x=\hat{A}_{m}^{I}\left(y_{m}-s_{m} y\right)+\hat{A}_{m}^{I} s_{m} y+x_{n}^{(0)}-\left(\hat{A}_{m}^{I} A_{n} r_{n} x+z_{n}^{(0)}\right),
$$

where $z_{n}^{(0)}$ is an element of $\operatorname{ker}\left(A_{n}\right)$ which depends on $r_{n} x$. On choosing $x_{n}^{(0)}=z_{n}^{(0)}$ and recalling that $y=A x$ we have

$$
x_{n}-r_{n} x=\hat{A}_{m}^{\prime}\left(y_{m}-s_{m} y\right)+\hat{A}_{m}^{\prime}\left(s_{m} A x-A_{n} r_{n} x\right) .
$$

Thus

$$
\left\|x_{n}-r_{n} x\right\| \leqslant\left\|\hat{A}_{m}^{I}\right\|\left\{\left\|y_{m}-s_{m} y\right\|+\left\|\delta_{n}^{A} x\right\|\right\}
$$

and the result follows. 
As an immediate consequence of this theorem we can determine the rate of convergence of the sequence $\left\{x_{n}-r_{n} x\right\}$ to zero. If $\left\|y_{m}-s_{m} y\right\|<C_{1} n^{-r_{1}},\left\|\delta_{n}^{A} x\right\|$ $<C_{2} n^{-r_{2}}$ and $\left\|\hat{A}_{m}^{\prime}\right\| \leqslant C_{3}+C_{4} \log n$ then from (4.1) we have that $\left\|x_{n}-r_{n} x\right\|<$ $(A+B \log n) n^{-r}$ where $r=\min \left(r_{1}, r_{2}\right)$ and the constants $A, B$ are independent of $n$.

Before being able to discuss the complete equation we need two further results concerning the dominant equation.

THEOREM 4.2. Suppose $\left\{A_{n}\right\}$ is $\alpha$-discretely consistent with $A$ on $\operatorname{dom}(A)$, then to every $y \in \operatorname{ran}(A)$ there exists an element $y_{m} \in \operatorname{ran}\left(A_{n}\right)$ such that the sequence $\left\{s_{m} y-y_{m}\right\}$ is $\alpha$-null.

Proof. Let $x \in \operatorname{dom}(A)$ be any element such that $A x=y$. Choose $x_{n}=r_{n} x$. Then $x_{n} \in \operatorname{dom}\left(A_{n}\right)$ and $y_{m}=A_{n} x_{n}$ is, by definition, in $\operatorname{ran}\left(A_{n}\right)$. Now $s_{m} y-y_{m}$ $=s_{m} A x-A_{n} r_{n} x=\delta_{n}^{A} x$, see (3.7). Since $\left\{A_{n}\right\}$ is $\alpha$-discretely consistent with $A$ on $\operatorname{dom}(A)$, the sequence $\left\{s_{m} y-y_{m}\right\}$ is $\alpha$-null.

Before stating the next theorem we require one further property for our operators $A_{n}, A$.

Definition 4.3. The sequence of operators $\left\{A_{n}\right\}$ is said to be compatible with the operator $A$ if $r_{n}\{\operatorname{ker}(A)\}=\operatorname{ker}\left(A_{n}\right)$, for each $n \geqslant n_{0}$.

Note that when $\kappa \leqslant 0$ it is trivially true that the sequence of operators $\left\{A_{n}\right\}$ is compatible with $A$.

THEOREM 4.4. Suppose

(i) $\left\{A_{n}\right\}$ is compatible with $A$,

(ii) $\left\{A_{n}\right\}$ is $\alpha$-discretely consistent with $A$ on $\operatorname{dom}(A)$,

(iii) $\left\{A_{n}\right\}$ is $\alpha$-stable.

Then the sequence $\left\{\hat{A}_{m}^{\prime}\right\}$ is $\alpha$-discretely consistent with $\hat{A}^{\prime}$ on $\operatorname{ran}(A)$.

Proof. Firstly let us write $X=\operatorname{ker}(A) \oplus X^{(1)}$ and $X_{n}=\operatorname{ker}\left(A_{n}\right)+X_{n}^{(1)}$. By (i) and Theorem 4.1 it follows that if $x^{(1)} \in X^{(1)}$ and $A x^{(1)}=y$, then there exists $x_{n}^{(1)} \in X_{n}^{(1)}$ such that $A_{n} x_{n}^{(1)}=y_{m}$ and the sequence $\left\{r_{n} x^{(1)}-x_{n}^{(1)}\right\}$ is $\alpha$-null.

Next, we need to show that for every $y \in \operatorname{ran}(A)$, the sequence $\left\{r_{n} \hat{A}^{\prime} y-\right.$ $\left.\hat{A}_{m}^{I} s_{m} y\right\}$ is $\alpha$-null. By Theorem 4.2, to each $y \in \operatorname{ran}(A)$ we can find a sequence $\left\{y_{m}\right\}$ with $y_{m} \in \operatorname{ran}\left(A_{n}\right)$ such that $\left\{s_{m} y-y_{m}\right\}$ is $\alpha$-null. Let $x^{(1)} \in X^{(1)}$ be that unique element such that $A x^{(1)}=y$ and $x_{n}^{(1)} \in X_{n}^{(1)}$ that unique element such 
that $A_{n} x_{n}^{(1)}=y_{m}$. Theri

$$
\begin{aligned}
\delta_{n}^{\hat{A}^{\prime} y} & =r_{n} \hat{A}^{I} y-\hat{A}_{m}^{I} s_{m} y \\
& =\left(r_{n} x^{(1)}-x_{n}^{(1)}\right)-\hat{A}_{m}^{I}\left(s_{m} y-y_{m}\right)
\end{aligned}
$$

Since each sequence on the right hand side is $\alpha$-null, the result follows.

\section{Convergence of discrete solutions for the complete equation}

When considering the complete equation we shall take as our starting point the fact that it is equivalent to a Fredholm integral equation (see (2.7)) and make use of the convergence theory for such equations. This equation has been extensively studied; a suitable form of convergence theorem has been given by Linz [5].

Theorem 5.1. Consider the equation $(I+J) x=g$ where $J$ is a compact operator on a Banach space $X$ into $X, I$ is the identity operator and $g$ is an element of $X$. Consider further the system of $n$ linear algebraic equations $\left(I_{n}+J_{n}\right) x_{n}=g_{n}$ where $g_{n} \in X_{n}$. If

(i) $(I+J)^{I}$ exists and is bounded,

(ii) $\left\{J_{n}\right\}$ is a consistent approximation to $J$,

(iii) $\lim _{n \rightarrow \infty}\left\|r_{n} g-g_{n}\right\|=0$,

(iv) $\left\{p_{n} J_{n}\right\}$ is collectively compact, then $\left\{I_{n}+J_{n}\right\}$ is stable and $\lim _{n \rightarrow \infty}\left\|r_{n} x-x_{n}\right\|=0$.

Proof. See Linz [5, Theorems 3 and 4], where definitions of consistency and stability are also given.

In comparing the statement of this theorem with equations (2.7) and (3.4) we see that we have $J=\hat{A}^{I} K$ and $J_{n}=\hat{A}_{m}^{I} K_{n}$. Since we are assuming that $k$ is Hölder continuous on $[-1,1] \times[-1,1]$ we have that $K$ is compact. Furthermore if we define $\left\|\hat{A}^{I}\right\|=\sup _{y \neq 0}\left(\left\|\hat{A}^{I} y\right\|_{\infty} /\|y\|_{H}\right)$, then $\hat{A}^{I}$ is bounded so that $\hat{A}^{I} K$ is compact as required by Theorem 5.1. Again, condition (i) of Theorem 5.1 expresses the fact that (2.7), and consequently (1.1), is solvable, which we certainly assume to be true. It remains to consider conditions (ii)-(iv) of Theorem 5.1. 
THEOREM 5.2. In addition to conditions (i)-(iii) of Theorem 4.4, let us suppose that $\left\{K_{n}\right\}$ is $\alpha$-discretely consistent with $K$ on a sub space $X^{(2)}$ say of $X$ which is such that

$$
X^{(2)}=\{x: K x \in \operatorname{ran}(A)\} \text { and } X^{(2)} \supseteq \operatorname{dom}(A) .
$$

Then $\left\{\hat{A}_{m}^{I} K_{n}\right\}$ is consistent with $\hat{A}^{\prime} K$ on $X^{(2)}$.

Proof. Since $J=\hat{A}^{\prime} K$ is a mapping from $X$ into itself and $J_{n}=\hat{A}_{m}^{\prime} K_{n}$ is a mapping from $X_{n}$ into $X_{n}$ we need to show that $\lim _{n \rightarrow \infty}\left\|\delta_{n}^{J} x\right\|=0$ for every $x \in X^{(2)}$, where $\delta_{n}^{J} x=r_{n} J x-J_{n} r_{n} x$. Now

$$
\begin{aligned}
\delta_{n}^{J} x & =r_{n} \hat{A}^{I}(K x)-\hat{A}_{m}^{I}\left(s_{m} K x-\delta_{n}^{K} x\right), \quad \text { by (3.7), } \\
& =\delta_{n}^{\hat{A}^{\prime}}(K x)+\hat{A}_{m}^{I} \delta_{n}^{K} x \text {, by (3.8). }
\end{aligned}
$$

By Theorem 4.4 the first sequence on the right is $\alpha$-rull. From the assumptions of this theorem, the second sequence on the right is $\alpha$-null so that $\left\{\delta_{n}^{J} x\right\}$ is $\alpha$-null and therefore null.

THEOREM 5.3. If in addition to conditions (i)-(iii) of Theorem 4.4 we suppose $y_{m}$ is such that the sequence $\left\{y_{m}-s_{m} y\right\}$ is $\alpha$-null, then condition (iii) of Theorem 5.1 is satisfied.

Proof. From (2.7) and (3.4) it is obvious that we have $g=\hat{A}^{\prime} y+x^{(0)}$ say where $x^{(0)} \in \operatorname{ker}(A)$, the space spanned by $b P_{\alpha-1}$, and $g_{n}=\hat{A}_{m}^{1} y_{m}+x_{n}^{(0)}$. Since we shall assume compatibility of $\left\{A_{n}\right\}$ with $A$ we shall choose $x_{n}^{(0)}=r_{n} x^{(0)}$ so that we need to show that $\left\{r_{n} \hat{A}^{I} y-\hat{A}_{m}^{I} y_{m}\right\}$ is $\alpha$-null. But

$$
r_{n} \hat{A}^{I} y-\hat{A}_{m}^{\prime} y_{m}=\delta_{n}^{\hat{A}^{\prime}} y+\hat{A}_{m}^{I}\left(s_{m} y-y_{m}\right) \text {, }
$$

and each sequence on the right hand side is $\alpha$-null, so that the result follows.

It finally remains to consider condition (iv) of Theorem 5.1.

Theorem 5.4. In addition to conditions (i)-(iii) of Theorem 4.4 let $\left\{q_{m} K_{n}\right\}$ be collectively compact on $X_{n}$ into $\operatorname{ran}(A)$. Then $\left\{p_{n} \hat{A}_{m}^{I} K_{n}\right\}$ is collectively compact on $X_{n}$ into $X$.

Proof. From (3.6)(iii) we can write

$$
p_{n} \hat{A}_{m}^{l} K_{n}=\left(p_{n} \hat{A}_{m}^{\prime} s_{m}\right)\left(q_{m} K_{n}\right)=M_{n}\left(q_{m} K_{n}\right)
$$

say, where $M_{n}=p_{n} \hat{A}_{m}^{I} s_{m}$ is a linear operator from $\operatorname{ran}(A)$ into $X$. We shall first show that to every element $y \in \operatorname{ran}(A)$, there exists $x \in \operatorname{dom}(A)$ such that $\lim _{n \rightarrow \infty} M_{n} y=x$. From Theorem 4.2 we know that under the given conditions 
we can find an element $y_{m} \in \operatorname{ran}\left(A_{n}\right)$ such that $\left\{s_{m} y-y_{m}\right\}$ is $\alpha$-null. If $A x=y$ and $A_{n} x_{n}=y_{m}$ then, by Theorem 4.1, $\left\{x_{n}\right\}$ can be chosen so that it converges $\alpha$-discretely to $x$. In particular it will converge globally to $x$, that is $\lim _{n \rightarrow \infty}\left\|x-p_{n} x_{n}\right\|=0$. Now

$$
M_{n} y-x=p_{n} \hat{A}_{m}^{I}\left(s_{m} y-y_{m}\right)+\left(p_{n} x_{n}-x\right)
$$

and since $\left\|p_{n}\right\| \leqslant p$ (see (3.5)(i)) it follows that $\lim _{n \rightarrow \infty} M_{n y}=x$.

Let $\left\{x_{n}\right\}$, with $x_{n} \in X_{n}$, be any bounded sequence. Since $\left\{q_{m} K_{n}\right\}$ is collectively compact on $X_{n}$ into $\operatorname{ran}(A)$ it follows that there exists a sub-sequence $\left\{x_{n_{k}}\right\}$ say, such that $\left\{q_{m_{k}} K_{n_{k}} x_{n_{k}}\right\}$ converges to an element $y$ say of $\operatorname{ran}(A)$. Furthermore we can always choose this subsequence so that $\left\|q_{m_{k}} K_{n_{k}} x_{n_{k}}-y\right\|<C k^{-\alpha_{1}}$ so that it is $\alpha$-convergent to $y$ when suitably relabelled. Consider

$$
p_{n_{k}} \hat{A}_{m_{k}}^{I} K_{n_{k}} x_{n_{k}}-x=M_{n_{k}}\left(q_{m_{k}} K_{n_{k}} x_{n_{k}}-y\right)+\left(M_{n_{k}} y-x\right) \text {. }
$$

We have already shown that the second sequence on the right hand side tends to zero as $k \rightarrow \infty$. Since $p_{n}$ and $s_{m}$ are uniformly bounded, since $\left\{A_{n}\right\}$ is $\alpha$-stable, and since we have shown that $\left\{q_{m_{k}} K_{n_{k}} x_{n_{k}}-y\right\}$ is $\alpha$-null it follows that the first sequence on the right hand side also tends to zero as $k \rightarrow \infty$. Thus from any bounded sequence $\left\{x_{n}\right\}$ we can extract a sub-sequence $\left\{p_{n_{k}} \hat{A}_{m_{k}}^{I} K_{n_{k}} x_{n_{k}}\right\}$ which is convergent to an element of $X$. Hence $\left\{p_{n} \hat{A}_{m}^{I} K_{n}\right\}$ is collectively compact on $X_{n}$ into $X$.

Having considered the conditions of Theorem 5.1, we can now state the principal result of this paper.

Theorem 5.5. Suppose $A$ is of index $\kappa$ and $y$ is such that $(A+K) x=y$ possesses a solution. Consider the sequence of linear algebraic equations $\left(A_{n}+K_{n}\right) x_{n}=y_{m}, m=n-\kappa$, where $A_{n}, K_{n}$ are $m \times n$ matrices, $A_{n}$ is of rank $=\min (m, n)$ and $y_{m} \in \operatorname{ran}\left(A_{n}\right)$. Suppose, in addition, that

(i) $\left\{A_{n}\right\}$ is compatible with $A$,

(ii) $\left\{A_{n}\right\}$ is $\alpha$-discretely consistent with $A$ on $\operatorname{dom}(A)$,

(iii) $\left\{A_{n}\right\}$ is $\alpha$-stable,

(iv) $\operatorname{ran}\left(K_{n}\right) \subseteq \operatorname{ran}\left(A_{n}\right)$,

(v) $\left\{K_{n}\right\}$ is $\alpha$-discretely consistent with $K$ on $X^{(2)} \supseteq \operatorname{dom}(A)$,

(vi) $\left\{q_{m} K_{n}\right\}$ is collectively compact on $X_{n}$ into $\operatorname{ran}(A)$,

(vii) $\left\{y_{m}-s_{m} y\right\}$ is $\alpha$-null.

Then, for all $n \geqslant n_{0}$, to each solution $x$ of $(A+K) x=y$ there corresponds a solution $x_{n}$ say of $\left(A_{n}+K_{n}\right) x_{n}=y_{m}$ such that the sequence $\left\{x_{n}\right\}$ converges both discretely and globally to $x$. Furthermore $\left(I_{n}+\hat{A}_{m}^{\prime} K_{n}\right)^{l}$ exists and is uniformly bounded. 
PROOF. This is an immediate consequence of the preceding theorems.

Finally, let us consider the rate of convergence of the approximate solutions to the solution of the complete equation. Let $B_{n}=I_{n}+\hat{A}_{m}^{I} K_{n}$, then from Theorem 5.5 we have that $\left\{B_{n}\right\}$ is stable. From (2.7) and (3.4), on choosing $x_{n}^{(0)}=r_{n} x^{(0)}$, we have

$$
\begin{aligned}
r_{n} x-x_{n} & =B_{n}^{I} B_{n} r_{n} x-B_{n}^{I} \hat{A}_{m}^{I} y_{m} \\
& =B_{n}^{I}\left\{r_{n} x+\hat{A}_{m}^{I}\left(K_{n} r_{n} x-y_{m}\right)\right\} \\
& =B_{n}^{I} \hat{A}_{m}^{I}\left\{A_{n} r_{n} x+K_{n} r_{n} x-y_{m}\right\}
\end{aligned}
$$

since $r_{n} x \in X_{n}^{(1)}$ and (see (3.2)) we have that $\hat{A}_{m}^{\prime} A_{n} x_{n}^{(1)}=x_{n}^{(1)}$ for every $x_{n}^{(1)} \in$ $X_{n}^{(1)}$. From definitions of $\delta_{n}^{A} x$ and $\delta_{n}^{K} x$ we find

$$
r_{n} x-x_{n}=-B_{n}^{I} \hat{A}_{m}^{I}\left\{\delta_{n}^{A} x+\delta_{n}^{K} x+\left(y_{m}-s_{m} y\right)\right\}
$$

from which it follows that

$$
\left\|r_{n} x-x_{n}\right\| \leqslant\left\|B_{n}^{I}\right\| \cdot\left\|\hat{A}_{m}^{I}\right\|\left\{\left\|\delta_{n}^{A} x\right\|+\left\|\delta_{n}^{K} x\right\|+\left\|y_{m}-s_{m} y\right\|\right\} .
$$

If, as in Section 4, we assume $\left\|y_{m}-s_{m} y\right\|<C_{1} n^{-r_{1}},\left\|\delta_{n}^{A} x\right\| \leqslant C_{2} n^{-r_{2}}$ and additionally $\left\|\delta_{n}^{K} x\right\| \leqslant C_{3} n^{-r_{3}}$ then since $\left\|B_{n}^{I}\right\|$ is uniformly bounded and $\left\|\hat{A}_{m}^{I}\right\| \leqslant C_{4}$ $+C_{5} \log n$ it follows that

$$
\left\|r_{n} x-x_{n}\right\| \leqslant(A+B \log n) n^{-r},
$$

where $r=\min \left(r_{1}, r_{2}, r_{3}\right)$ and $A, B$ are independent of $n$.

For an example of the application of these results see Elliott [3].

\section{References}

[1] M. L. Dow and David Elliott, "The numerical solution of singular integral equations over $(-1,1)$ ", SIAM J. Numer. Anal. 16 (1979), 115-134.

[2] David Elliott, "Orthogonal polynomials associated with singular integral equations having a Cauchy kernel", Univ. of Tasmania Maths. Dept. Tech. Rep. No. 144 (1980).

[3] David Elliott, "The classical collocation method for singular integral equations", Univ. of Tasmania Maths. Dept. Tech. Rep. No. 145 (1980).

[4] F. D. Gakhov, Boundary value problems (Oxford: Pergamon Press, 1966).

[5] Peter Linz, "A general theory for the approximate solution of operator equations of the second kind", SIAM J. Numer. Anal. 14 (1977), 543-554.

[6] Peter Linz, “An analysis of a method for solving singular integral equations”, BIT 17 (1977), 329-337.

[7] N. I. Muskhelishvili, Singular integral equations (Gröningen: P. Noordhoff, 1953).

[8] B. Noble, Applied linear algebra (Englewood Cliffs N.J.: Prentice-Hall, 1969). 
[9] B. Noble, "Error analysis of collocation methods for solving Fredholm integral equations", in Topics in numerical analysis (ed. J. J. H. Miller) (New York: Academic Press, 1973), 211-232.

[10] Friedrich Stummel, "Diskrete konvergenz linearer operatoren I", Math. Ann. 190 (1970), 45-92.

\section{Mathematics Department}

University of Tasmania

Box 252C, G.P.O.

Hobart, Tasmania, 7001 